\title{
28 Research Square \\ Predictive Value of a Five-biomarker Signature to Diagnose Active Pulmonary Tuberculosis Patients
}

Jiang Zeng

Ganzhou Fifth People's Hospital

Qianting Yang

Shenzhen Third People's Hospital

Dechang Xu

Ganzhou Fifth People's Hospital

Qi Chen

Shenzhen Third People's Hospital

Kaisong Huang

Shenzhen University

Yi Cai

Shenzhen University

Youchao Dai

Shenzhen University

Xie Hai

Shenzhen University

Zhong Zeng

Ganzhou Fifth People's Hospital

Xiangwen Gong

Ganzhou Fifth People's Hospital

Haiying Peng

Ganzhou Fifth People's Hospital

Guofang Deng

Shenzhen Third People's Hospital

Xinchun Chen

Shenzhen University

Huihua Zhang ( $\nabla$ hh_zhang@szu.edu.cn )

Shenzhen University https://orcid.org/0000-0002-3187-4291

Hui Liu

Ganzhou Fifth People's Hospital 
Keywords: pulmonary tuberculosis, pneumonia, acid-fast bacilli staining, Interferon-gamma release assays, multivariate risk model

Posted Date: January 20th, 2021

DOI: https://doi.org/10.21203/rs.3.rs-148655/v1

License: (c) (1) This work is licensed under a Creative Commons Attribution 4.0 International License. Read Full License 


\section{Abstract}

Background: To improve the diagnosis accuracy of active pulmonary tuberculosis (TB) from pneumonia (PN) in low income and inadequate facilities areas is one of the biggest problems facing public health today. Most TB patients are difficult to diagnose, especially those who are acid-fast bacillus smearnegative (AFB') but IGRA®.TB test positive (IGRA ${ }^{+}$). Thus, we aim to develop a low-cost and rapid risk model for the diagnosis of TB patients with $\mathrm{AFB}^{-} \mathrm{IGRA}^{+} \mathrm{TB}$ from $\mathrm{PN}$.

Methods: We retrospectively analyzed 41 laboratory variables of 204 AFB- IGRA $^{+}$TB and 156 PN participants. Candidate variables were identified by t-statistic test and univariate logistic model. The logistic regression analysis was used to construct the multivariate risk model and nomogram with internal and external validation.

Results: There were differential correlations between variable pairs in AFB' IGRA ${ }^{+}$TB and PN. We found several significant variables in TB compared with PN. Among them, uric acid (UA) was up-elevated, acting as a protective factor with an odds ratio $(O R)<1$. By integrating five variables, including Age, UA, albumin (ALB), hemoglobin ( $\mathrm{Hb}$ ) and white blood cell counts (WBC), we constructed a multivariate risk model with a concordance index (C-index) of 0.7 (95\% Cl: $0.61,0.8)$. Nomogram showed that $\mathrm{UA}$ and $\mathrm{Hb}$ had the protection effect, while Age, WBC and ALB acted as risk factors on TB occurrence. Internal and external validation revealed a good agreement between nomogran prediction and actual observations.

Conclusions: Differential correlations existed between variable pairs in AFB- IGRA' integration of five biomarkers (Age, UA, ALB, Hb and WBC) can be used to predict TB in AFB' IGRA ${ }^{+}$ clinical samples from PN.

\section{Background}

Tuberculosis remains a severe global public health problem, especially in developing countries[1]. As of 2018, Jiangxi province, China, reported 34,000 new cases of tuberculosis each year, with a reported incidence of 70.83/100,000, ranking second among infectious diseases for a long time. How to reduce the tuberculosis epidemic in the province is a major issue in economic and social development.

Detection of Mycobacterium tuberculosis (Mtb) DNA using Gene Xpert MTB/RIF assay is more sensitive and rapid for diagnosing TB and rifampicin resistance. However, due to its costs, environmental limitations and supplement, it is difficult to carry out screening in a low-income rural area[2, 3]. Interferongamma release assay (IGRAs) is commonly used in the diagnostic workup of Mtb, but distinguish poorly between active TB (TB) and latent tuberculosis infection (LTBI) $[4,5]$. In addition, the symptoms of tuberculosis patients are very similar to those of pneumonia, especially when most tuberculosis patients have no sputum specimens, or are Mtb smear/culture-negative, which makes diagnosis more intractable. Therefore, the development of new detection methods to improve diagnostic accuracy of sputum smearnegative tuberculosis is still important. 
Host markers including secreted molecules in blood have been reported as novel candidate markers to distinguish TB from LTBI, such as interferon-gamma inducible protein ten KD (IP-10)[6], Interleukin 2 (IL-2) [7], IL-6 [8],C-reactive proteins (CRP) [9] and Vascular endothelial growth factor (VEGF)[8]. However, the reported biomarkers' diagnostic performances could not be applied in low-income areas due to their technical, instrumentation or high price issues. To address this problem, the present study retrospectively analyzed the differences in routinely monitored laboratory variables in blood test between $\mathrm{AFB}^{-}$IGRA $^{+} \mathrm{TB}^{-}$ and $\mathrm{PN}$ and build a risk model to differentiate $\mathrm{AFB}^{-} \mathrm{IGRA}^{+} \mathrm{TB}$ from $\mathrm{PN}$, and validated its application by an external independent cohort.

\section{Methods}

\section{Study design and subject oversight}

The patients provided a full medical history, participated in regular physical examinations and underwent routine investigations, including HIV serology, chest radiography, IGRAs and microbiological sputum examination, where possible. All participants in this study were excluded from HIV infection. Pneumonia (PN) cases referred to upper or lower respiratory tract infections by viral or non-Mtb bacterial pathogens, although no attempts to identify the organisms by bacterial or viral cultures were made. Acid-fast bacilli (AFB)-negative active pulmonary TB (TB) participants were no sputum or negative smear and negative Mtb, but chest computed tomographic (CT) scans or chest X-ray evidence and symptoms responding to TB treatment.

The involved participants were divided into two groups: the discovery cohort and the external validation cohort. For the discovery cohort, participants were enrolled in Ganzhou Fifth Hospital (Ganzhou, China) between August 2018 and August 2020, including 748 AFB-negative TB participants and 531 PN participants. As the predefined goal was to assess the ability of laboratory biomarkers to distinguish IGRA-positive patients presenting with AFB-negative TB, 287 participants with IGRA-positive TB were subjected for further analysis (AFB- IGRA ${ }^{+} \mathrm{TB}$ ). To analyze more biomarkers, participants with over $50 \%$ laboratory data missing were excluded. Totally 89 TB and 38 PN participants with recorded biomarker values were used to construct a risk model after statistical analysis. The external validation cohort of 134 participants in the study was collected from Shenzhen Third People's Hospital (Shenzhen, China) from June 2018 and June 2019 (Fig. 1). Among them, 15 were excluded due to missing variables, consisting 77 AFB- IGRA $^{+}$TB and 42 PN participants.

\section{Data collection}

The medical records of all participants were reviewed by experienced TB clinicians, including medical history, symptoms, clinical signs, microbiological test, laboratory finding, chest CT chest x-ray, and treatment measures. Forty-one laboratory biomarkers were assessed by differential statistics and odds ratio (OR) calculation for variable selection (Additional file 2: Table S2).

\section{Statistical analyses}


For laboratory results, continuous variables were preprocessed by log2-transformation before data analysis. Laboratory variables were compared using moderated t-statistics in the R package "limma", which is suitable for data that may not be normally distributed[10, 11]. P-values were adjusted by the false discovery rate (FDR). Variables between two conditions were defined as statistically significant when the FDR was $<0.2[12]$.

A generalized linear model ( $\mathrm{glm}$ ) was used to calculate odds ratio (OR) for each laboratory biomarker. The regression coefficient of the glm was regarded as the log OR. Variables which FDR was $<0.2$ or at a statistically significant level $(p$-value $<0.05)$ in univariate $\mathrm{glm}$ analysis were candidates for construction of a multivariate logistic regression risk model (Irm) and nomogram by R package "rms", and the finial variables were determined using Akaike's information criterion (AIC) as a stopping rule. The goodness of fit of multivariate risk model Irm was calculated by Hosmer and Lemeshow $\mathrm{C}$ statistic. The performance

of nomogram was evaluated by the concordance index (C-index) and assessed by comparing nomogrampredicted vs actual observation, and bootstraps with 1000 resamples to decrease the overfit bias were applied to calibration[13, 14].

Differences in the correlations of biomarker pairs between TB and PN were analyzed by R package "DGCA". DGCA transforms sample correlation coefficients to z-scores and uses differences in z-scores to calculate p-values of differential correlation between gene pairs[15]. Package "pheatmap" was used to draw a correlation heatmap between two conditions. All analyses and figures were generated in $\mathrm{R}$ version 4.0.3 (https://www.r-project.org/) and arranged for publishing using Photoshop CS3 (Adobe, San Diego, CA, USA).

\section{Results}

\section{Baseline characteristics of AFB- IGRA ${ }^{+}$TB and PN participants}

The characteristics of the AFB- IGRA' ${ }^{+}$TB and PN participants were shown in Additional file 1: Table S1. No significant age and gender differences were found in the first cohort. Males made up the majority in both two cohorts. Clinical types of TB participants included 162 infiltrative pulmonary tuberculosis, 19 cavitary pulmonary tuberculosis, 8 secondary pulmonary tuberculosis, and 15 tuberculous pleurisy and empyema. Only 44 and 23 participants have received TB-DNA and TB-antibody examiniation, yielding $9.09 \%(4 / 44)$ and $13.04 \%(3 / 23)$ positive rates respectively.

\section{Differential laboratory biomarkers between AFB- IGRA' TB and PN}

To gain more insights into the overall change in correlation structure in laboratory variables between $\mathrm{AFB}^{-} \mathrm{IGRA}^{+} \mathrm{TB}$ and $\mathrm{PN}$, we used DGCA to visualize all variable pair correlations in both conditions (Fig. 2A). Variables in this heatmap were ordered by their median z-score correlation difference with all of the other variables, without the restriction to positive correlations. By quantified the difference in correlation between all variable pairs using permutation testing[15], we found a lower correlation between 
most variable pairs in TB than PN (mean difference in z-score $(\mathrm{dz})=0.11, p=0.04$ ), suggesting that the TB group had a more coordinated perturbation of profile than the PN group.

We further explored the significant variables between the two conditions. Using t-statistics and setting an FDR $<0.2,13$ variables with marked differences were identified (Table 1, Additional file 2: Table S2). Only uric acid (UA) was up-regulated (foldchange $>1.2$ ). Five variables, including aspartate aminotransferase (AST), total bile acid (TBA), triglyceride (TG), alanine aminotransferase (ALT) and glutamyl transpeptidase (GGT), were down-regulated (foldchange < 0.83) in TB compared with PN (Fig. 2B).

Table 1

Differential variables and OR values in $\mathrm{AFB}^{-} \mathrm{IGRA}^{+} \mathrm{TB}$ compared with $\mathrm{PN}$ group.

\begin{tabular}{|c|c|c|c|c|c|c|c|}
\hline Variables & p-value & FDR & Foldchange & OR & p-value of OR & $2.5 \% \mathrm{Cl}$ & $97.5 \% \mathrm{Cl}$ \\
\hline UA & $6.82 \mathrm{E}-06$ & 2.73E-04 & 1.30 & 0.36 & $2.49 \mathrm{E}-05$ & 0.22 & 0.57 \\
\hline GLU & $6.94 \mathrm{E}-04$ & 0.01 & 0.88 & 4.26 & $1.45 \mathrm{E}-03$ & 1.82 & 10.93 \\
\hline AST & 8.54E-04 & 0.01 & 0.82 & 1.78 & 1.47E-03 & 1.26 & 2.56 \\
\hline TBA & 5.90E-03 & 0.06 & 0.66 & 1.59 & 0.01 & 1.14 & 2.30 \\
\hline TG & $1.45 \mathrm{E}-02$ & 0.11 & 0.82 & 2.66 & 0.02 & 1.20 & 6.65 \\
\hline RBC & 0.02 & 0.11 & 1.11 & 0.28 & 0.02 & 0.09 & 0.72 \\
\hline ALT & 0.02 & 0.13 & 0.82 & 1.31 & 0.03 & 1.03 & 1.67 \\
\hline $\mathrm{Hb}$ & 0.03 & 0.13 & 1.05 & 0.37 & 0.03 & 0.15 & 0.89 \\
\hline GGT & 0.03 & 0.14 & 0.72 & 1.33 & 0.04 & 1.02 & 1.74 \\
\hline ALP & 0.04 & 0.14 & 0.86 & 1.92 & 0.05 & 1.02 & 3.71 \\
\hline LDH & 0.04 & 0.14 & 0.83 & 1.60 & 0.06 & 1.01 & 2.71 \\
\hline ALB & 0.04 & 0.14 & 1.05 & 0.40 & 0.05 & 0.16 & 0.98 \\
\hline WBC & 0.06 & 0.19 & 0.93 & 1.49 & 0.06 & 0.98 & 2.29 \\
\hline $\begin{array}{l}\text { FDR, false } \\
\text { glucose. A } \\
\text { ALT, alanin } \\
\text { phosphata }\end{array}$ & $\begin{array}{l}\text { scovery ra } \\
\text { aspartate } \\
\text { aminotran } \\
\text { LDH, lac }\end{array}$ & $\begin{array}{l}\text { threshold } \\
\text { minotrans } \\
\text { rase. Hb, } \\
\text { e dehydros }\end{array}$ & $\begin{array}{l}\text { 2. OR, odds } r \\
\text { ase. TBA, tota } \\
\text { noglobin. GG } \\
\text { lase. ALB, alb }\end{array}$ & $\begin{array}{l}\text { ylutam } \\
\text { hin. W }\end{array}$ & $\begin{array}{l}\text { nfidence interv } \\
\text { TG, triglycerid } \\
\text { transpeptidas } \\
\text {, white blood }\end{array}$ & $\begin{array}{l}\text { UA, uric } \\
\text { BC, red } \\
L \text { LP, alkal }\end{array}$ & $\begin{array}{l}\text { d. GLU, } \\
\text { od cell. }\end{array}$ \\
\hline
\end{tabular}

Z-transformed correlation in these 13 variables also revealed differential connectivity between $\mathrm{AFB}^{-}$ IGRA ${ }^{+}$TB and PN, for example, pair hemoglobin $(\mathrm{Hb})$ and UA had no significant correlation ( $p$-value> 0.05 ) in TB, while had positive correlation [p-value $<0.05$, $\operatorname{Corr}(\rho)>0.3$ ] in PN; pair white blood cell counts (WBC) and UA had no significant correlation in TB ( $p$-value $>0.05)$, while had negative correlation in PN 
[p-value $<0.05$, Corr $(\rho)=-0.3$, Fig. $2 C]$. These data revealed variable expression differences between two conditions, and some variables might be useful to distinguish $\mathrm{AFB}^{-} \mathrm{IGRA}^{+} \mathrm{TB}$ from PN.

In order to find the odds that TB would progress or not given exposure to these laboratory variables, odds ratio (OR) was assessed for each variable by a univariate general linear model. We identified 11 variables significantly associated with TB progression ( $p$-value $<0.05$, Table 1 ). Among them, four variables indicated the protective effect TB progression ( $O R<1)$, including $U A$, red blood cell $(R B C), H b$ and albumin (ALB); while seven variables showed as risk factors in TB progression $(\mathrm{OR}>1)$.

\section{Multivariate risk model to predict TB progression probability}

Combining with the results above, and using $\mathrm{AIC}$ as a stopping rule, we finally selected five laboratory variables (Age, UA, ALB, $\mathrm{Hb}$ and $\mathrm{WBC}$ ) to develop a multivariate risk model with $89 \mathrm{AFB}^{-}$IGRA $^{+} \mathrm{AFB}$ and $38 \mathrm{PN}$ participants. Figure 2D represented the expression distribution of variables in both groups, showing more unbalance in $\mathrm{PN}$ than $\mathrm{AFB}^{-} \mathrm{IGRA}^{+} \mathrm{TB}$. The risk model yielded a C-index of $0.7(95 \% \mathrm{Cl}$ : $0.61,0.8$, Fig. $3 \mathrm{~A}$ black line), with $p$-value $=0.01$ (Chi-square test). The calibration plot revealed a moderate agreement between the risk model prediction and the actual observation (Fig. 3B, p-value = 0.18 , Hosmer-Lemeshow test).

Using the nomogram, we mapped the values for each variable to points on a scale axis ranging from 0 to 10. With a corresponding number of point assigned to given magnitudes of the variables, the risk probability was calculated by corresponding cumulative point score for all the variables[16]. We found that UA had the most protective effect on TB progression, followed by $\mathrm{Hb}$; while Age, WBC and ALB acted as risk factors (Fig. 3C).

\section{The Multivariate risk performed well in an independent validation cohort}

Next, we prospectively collected an external validation cohort of 134 participants, of whom 15 were excluded due to missing variables, consisting $77 \mathrm{AFB}^{-}$IGRA $^{+}$TB and 42 PN participants. The total points of each participant in the external cohort were calculated according to the established nomogram, and then used to evaluate the performance of the nomogram. The C-index of nomogram for predicting external cohort was 0.77 (95\% Cl: 0.68, 0.86, Fig. 3A red line). The calibration plot also showed good agreement between the prediction by nomogram and actual observation (Fig. 3D) with a p-value of 0.13 by Hosmer-Lemeshow test.

\section{Discussion}

In the study, different profiles were analyzed between $\mathrm{AFB}^{-}$IGRA ${ }^{+} \mathrm{TB}$ and PN from several aspects, and five laboratory variables (Age, UA, ALB, $\mathrm{Hb}$ and WBC) were selected to construct a multivariate risk model and nomogram. Internal validation and calibration plot showed moderate agreement between nomogram probability and actual observation, with a C-index of 0.7 (95\% Cl: $0.61,0.8)$. We achieved a similar result in an external validation cohort (C-index: $0.77,95 \% \mathrm{Cl}$ : 0.68, 0.86). These findings indicated that five 
laboratory variables can be used to predict TB disease probability, when a clinical sample was AFBnegative and IGRA-positive.

It was reported that TB patients intended to display increased levels of C-reaction protein (CRP), erythrocyte sedimentation rate (ESR), $\mathrm{UA}$ and low levels of $\mathrm{Hb}[17]$. Anemia is a common comorbidity in TB with a decreased level of $\mathrm{Hb}$. Prevalence of anemia in TB patients ranged between $32 \%$ and $96 \%[18]$, most of which were due to anemia of inflammation[19,20]. Previous evidence had showed an unambiguous relationship between anemia, iron redistribution and TB susceptibility[21], moreover anemia was correlated with poor clinical prognoses and mortality after TB diagnosis[20].

The anti-tuberculous agent Pyrazinamide (PZA) and ethambutol could increase serum UA level through decreasing in UA excretion and the production of UA by decreasing renal clearance, respectively[22, 23]. Increased UA level was observed in $28.2 \%$ of men and $37.5 \%$ of women prior to chemotherapy, and more often during the first 2 months of treatment both in men and women, which suffered from multiple drug resistant pulmonary TB[24]. In our study, serum UA showed significantly higher level in TB (FDR $<0.001)$, with an OR value of 0.36 ( $p$-value $<2.5 \mathrm{e}-05)$ compared with PN (Table 3).

Reduced plasma ALB concentrations have been reported in TB[25] and might be used as a diagnostic and prognostic marker in pretreated HIV and TB patients. Hypoalbuminemia was associated with an increased risk of mortality in patients with tuberculosis and serum albumin concentrations $<3.2 \mathrm{~g} / \mathrm{dL}$ were associated with $85 \%$ specificity[26]. WBC was significantly increased in TB patients compared with healthy controls, and the WBC decreased significantly during TB treatment [27, 28]. In our study, WBC was statistically significant and a significantly risk factor (OR: 1.49), but no higher counts in foldchange compared with PN (Table 3).

To predict the risk of TB for each $\mathrm{AFB}^{-} \mathrm{IGRA}^{+}$patient, we used a nomogram to provide a more accurate profile. With five variables, nomogram had a well predictive accuracy with a C-index of 0.7 , of which UA had a most protection contribution on TB progression (OR: 0.36, p-value $<0.01$, data not shown), indicating that it might be a specific protection factor on TB patients in our data. External validation is essential to confirm that it can be applied to patients outside of the cohort. Thus, we collected a second participant cohort from another center and tested them on the nomogram, and the result showed a good consistent with actual observation (C-index of 0.77 ).

The present study still had several limitations: first, in low-income and setting area, not all patients received all routine laboratory tests, leading to too many missing values in the first cohort of participants. In order to analyze more biomarkers, participants with over $50 \%$ of missing data were excluded, leaving 41 laboratory variables and a small number of participants left (89 AFB- IGRA' TBA and 38 PN). Second, though internal and external validation met good performance, further investigations were required to optimize the nomogram in larger cohorts and in more types of pulmonary tuberculosis. After improvement, we anticipated this model might help clinicians to reduce the cost and time to diagnose AFB $^{-}$IGRA ${ }^{+}$TB in low-income, high-burdened and resource-constrained setting rural area. 


\section{Conclusion}

The study had identified a five-variable signature in distinguish $\mathrm{AFB}^{-}$IGRA $^{+}$TB from PN patients. A risk model was built to differentiate $\mathrm{AFB}^{-} \mathrm{IGRA}^{+} \mathrm{TB}$ from $\mathrm{PN}$, and validated in an external independent cohort, which could be applied in low-income and resource-constrained setting rural areas.

\section{List Of Abbreviations}

TB, tuberculosis.

PN, pneumonia.

AFB, acid fast bacilli staining.

IGRA, IGRA®.TB test.

FDR, false discovery rate.

OR, odds ratio.

$\mathrm{Cl}$, confidence interval.

UA, uric acid.

GLU, glucose.

AST, aspartate aminotransferase.

TBA, total bile acid.

TG, triglyceride.

RBC, red blood cell.

ALT, alanine aminotransferase.

$\mathrm{Hb}$, hemoglobin.

GGT, glutamyl transpeptidase.

ALP, alkaline phosphatase.

LDH, lactate dehydrogenase.

ALB, albumin.

WBC, white blood cell. 
LDL, low density lipoprotein.

ADA, adenosine deaminase.

GLB, globulin.

HDL, high density lipoprotein.

LYMPH, lymphocyte count.

$\mathrm{Na} \%$, sodium.

NEU\%, neutrophil ratio.

CHE, cholinesterase.

CRP, c-reaction protein.

TBIL, total bilirubin.

CREA, creatinine.

IBIL, indirect bilirubin.

NEU, Neutrophil count.

PLT, platelet.

CL\%, chloridion.

TC, total cholesterol.

BUN, blood urea nitrogen.

$\mathrm{CA} \%$, calcium.

$T P$, total protein.

LYMPH\%, lymphocyte ratio.

PCT, procalcitonin.

DBIL, direct bilirubin.

$\mathrm{K} \%$, kalium.

AST/ALT, ratio of AST and ALT. 
$\mathrm{D} / \mathrm{T}$, ratio of DBIL and TBIL.

\section{Declarations}

\section{Contributions}

Xinchun Chen, Dechang Xu and Hui Liu designed the study. Jiang Zeng, Qi Chen, Youchao Dai, Zhong Zeng, Xiangwen Gong, Haiying Peng and Guofang Deng recruited clinical records and assessed the participants. Huihua Zhang, Qianting Yang, Kaisong Huang , Yi Cai and Hai Xie managed statistical analyses and drafted the manuscript. All authors were involved in critically revising and providing finial manuscript approval.

\section{Acknowledgments}

We thank the staff of the Ganzhou Fifth Hospital and Shenzhen Third People's Hospital for facilitating access to the relevant medical records.

\section{Funding}

This work was supported by Natural Science Foundation of Jiangxi Province (20202BAB206059).

\section{Ethics declarations}

\section{Ethical approval and consent to participate}

The study protocol was approved by the Ethics Committee of Ganzhou Fifth Hospital and Shenzhen Third People's Hospital to allow retrospective access to patients' records and files. Written informed consent was waived by the Ethics Commission as this was an observational and retrospective analysis.

\section{Consent for publication}

Not applicable.

\section{Available of data and materials}

The clinical data used and/or analyzed during the current study are available from the corresponding author on reasonable request.

\section{Competing interests}

The authors declare no conflict of interest.

\section{References}

1. Organization WH: Global tuberculosis report 2020. 2020. 
2. Trébucq A, Da. E, Cy. C, A. VD, Ad. H, F. B, A. D, Pi. F, Sm. G, I. M et al: Xpert® MTB/RIF for national tuberculosis programmes in low-income countries: when, where and how? Int $J$ Tuberc Lung Dis 2011, 15(12):1567-1572.

3. Walzl G, McNerney R, du Plessis N, Bates M, McHugh TD, Chegou NN, Zumla A: Tuberculosis: advances and challenges in development of new diagnostics and biomarkers. Lancet Infect Dis 2018, 18(7):e199-e210.

4. Lim WS: From latent to active TB: are IGRAs of any use? Thorax 2016, 71(7):585-586.

5. Auguste P, Tsertsvadze A, Pink J, Court R, McCarthy N, Sutcliffe P, Clarke A: Comparing interferongamma release assays with tuberculin skin test for identifying latent tuberculosis infection that progresses to active tuberculosis: systematic review and meta-analysis. BMC Infect Dis 2017, 17(1):200.

6. Chegou NN, J. H, G. W, C. L, Ruhwald M: Beyond the IFN-y horizon: biomarkers for immunodiagnosis of infection with Mycobacterium tuberculosis. Eur Respir J 2014, 43(5):1472-1486.

7. Mamishi S, B. P, M. T, Pa. R, E. T, Aa. K, Mahmoudi S: Diagnostic accuracy of IL-2 for the diagnosis of latent tuberculosis: a systematic review and meta-analysis. Eur J Clin Microbiol Infect Dis 2014, 33(12):2111-2119.

8. Ahmad R, Xie L, Pyle M, Suarez M, Broger T, Steinberg D, Ame SM, Lucero MG, Szucs MJ, MacMullan $\mathrm{M}$ et al: A rapid triage test for active pulmonary tuberculosis in adult patients with persistent cough. . Sci Transl Med 2019, 11(514):eaaw8287.

9. Yoon C, Semitala FC, Atuhumuza E, Katende J, Mwebe S, Asege L, Armstrong DT, Andama AO, Dowdy DW, Davis JL et al: Point-of-care C-reactive protein-based tuberculosis screening for people living with HIV: a diagnostic accuracy study. Lancet Infect Dis 2017, 17(12):1285-1292.

10. Smyth GK: Linear models and empirical bayes methods for assessing differential expression in microarray experiments. Statistical Applications in Genetics and Molecular Biology 2004, 3(1).

11. Smyth GK: limma: Linear Models for Microarray Data. In: Bioinformatics and Computational Biology Solutions Using R and Bioconductor. edn. Edited by Gentleman R, Carey VJ, Huber W, Irizarry RA, Dudoit S. New York, NY: Springer New York; 2005: 397-420.

12. Capanu M, Seshan VE: False discovery rates for rare variants from sequenced data. Genet Epidemiol 2015, 39(2):65-76.

13. Tang L-Q, Li C-F, Li J, Chen W-H, Chen Q-Y, Yuan L-X, Lai X-P, He Y, Xu Y-X-X, Hu D-P et al: Establishment and Validation of Prognostic Nomograms for Endemic Nasopharyngeal Carcinoma. JNCI: Journal of the National Cancer Institute 2015, 108(1):291.

14. Jang JY, T. P, S. L, Y. K, Sy. L, Sw. K, Sc. K, Fau. SK, M. Y, T. H et al: Proposed Nomogram Predicting the Individual Risk of Malignancy in the Patients With Branch Duct Type Intraductal Papillary Mucinous Neoplasms of the Pancreas. Ann Surg 2017, 266(6):1062-1068.

15. McKenzie AT, Katsyv I, Song WM, Wang M, Zhang B: DGCA: A comprehensive R package for Differential Gene Correlation Analysis. BMC Syst Biol 2016, 10(1):106. 
16. Balachandran VP, Gonen M, Smith JJ, DeMatteo RP: Nomograms in oncology: more than meets the eye. Lancet Oncol 2015, 16(4):e173-180.

17. Gil-Santana L, Cruz LAB, Arriaga MB, Miranda PFC, Fukutani K, Silveira-Mattos PS, Silva EC, Oliveira MG, Mesquita EDD, Rauwerdink A et al: Tuberculosis-associated anemia is linked to a distinct inflammatory profile that persists after initiation of antitubercular therapy. Sci Rep 2019, 9(1):1381.

18. Barzegari S, Afshari M, Movahednia M, Moosazadeh M: Prevalence of anemia among patients with tuberculosis: A systematic review and meta-analysis. India J Tuberc 2019, 66(2):299-307.

19. Minchella PA, Donkor S, Owolabi O, Sutherland JS, McDermid JM: Complex anemia in tuberculosis: the need to consider causes and timing when designing interventions. Clin Infect Dis 2015, 60(5):764-772.

20. Minchella PA, Donkor S, Owolabi O, Sutherland JS, McDermid JM: Complex Anemia in Tuberculosis: The Need to Consider Causes and Timing When Designing Interventions. Clinical Infectious Diseases 2015, 60(5):764-772.

21. McDermid JM, Bj. H, M. vdS, Av. H, Hc. W, A. J, Prentice AM: Host iron redistribution as a risk factor for incident tuberculosis in HIV infection: an 11-year retrospective cohort study. BMC Infect Dis 2013, 13:48.

22. Moriwaki Y: Effects on Uric Acid Metabolism of the Drugs except the Antihyperuricemics. Journal of Bioequivalence \& Bioavailability 2014, 6(1):010-017.

23. Thumamo Pokam B, Enoh J, Eyo A-A, Umoh N, Guemdjom P: Uric acid levels in patients on antituberculosis drugs in the southwest Region of Cameroon. International Journal of Mycobacteriology 2018, 7(1):89-91.

24. R.Y. A, O.G. K, E.S. C, V.S. O: Level of uric acid in blood serum of new pulmonary tuberculosis patients with multiple drug resistance. Tuberculosis and Lung Diseases 2017, 95(4):31-36.

25. Bisaso KR, Owen JS, Ojara FW, Namuwenge PM, Mugisha A, Mbuagbaw L, Luboobi LS, Mukonzo JK: Characterizing plasma albumin concentration changes in TB/HIV patients on anti retroviral and anti tuberculosis therapy. In Silico Pharmacol 2014, 2(1):3.

26. Alvarez-Uria G, M. M, R. P, Naik PK: Diagnostic and Prognostic Value of Serum Albumin for Tuberculosis in HIV Infected Patients Eligible for Antiretroviral Therapy: Datafrom an HIV Cohort Study in India. Bioimpacts 2013, 3(3):123-128.

27. Rohini K, Surekha Bhat M, Srikumar PS, Mahesh Kumar A: Assessment of Hematological Parameters in Pulmonary Tuberculosis Patients. Indian J Clin Biochem 2016, 31(3):332-335.

28. Chedid C, Kokhreidze E, Tukvadze N, Banu S, Uddin MKM, Biswas S, Russomando G, Acosta CCD, Arenas R, Ranaivomanana PPR et al: Association of baseline white blood cell counts with tuberculosis treatment outcome: a prospective multicentered cohort study. International Journal of Infectious Diseases 2020, 100:199-206.

\section{Figures}




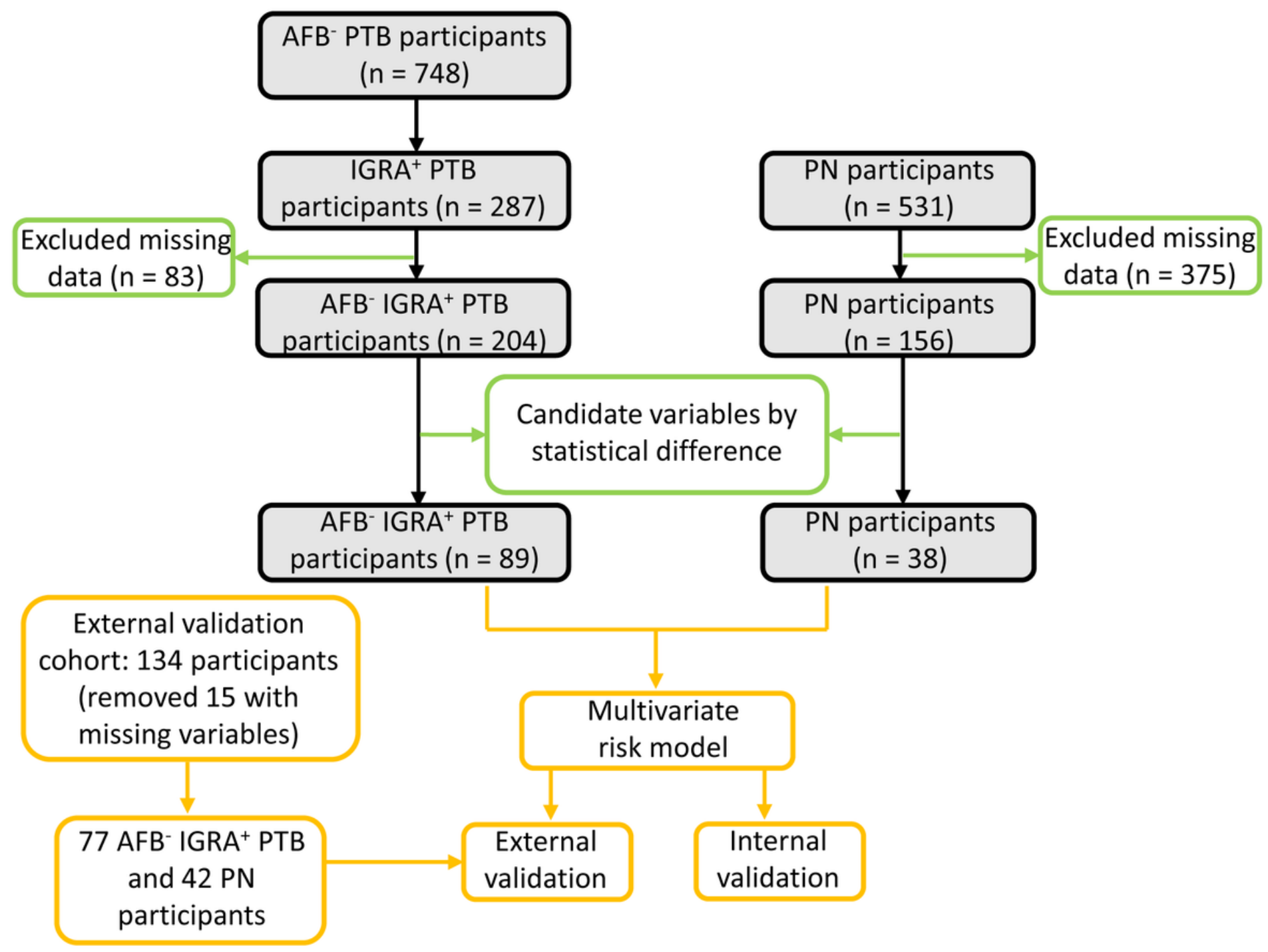

Figure 1

Overview of study design and analysis workflow. A total of 748 AFB- TB and 531 PN participants were prospectively evaluated. Individuals with over $50 \%$ of clinical data missing were excluded. Candidate variables were filtered by statistical differences. 89 AFB- IGRA+ TB participants and 38 PN participants with candidate variables were finally included in multivariate risk model construction. 
a

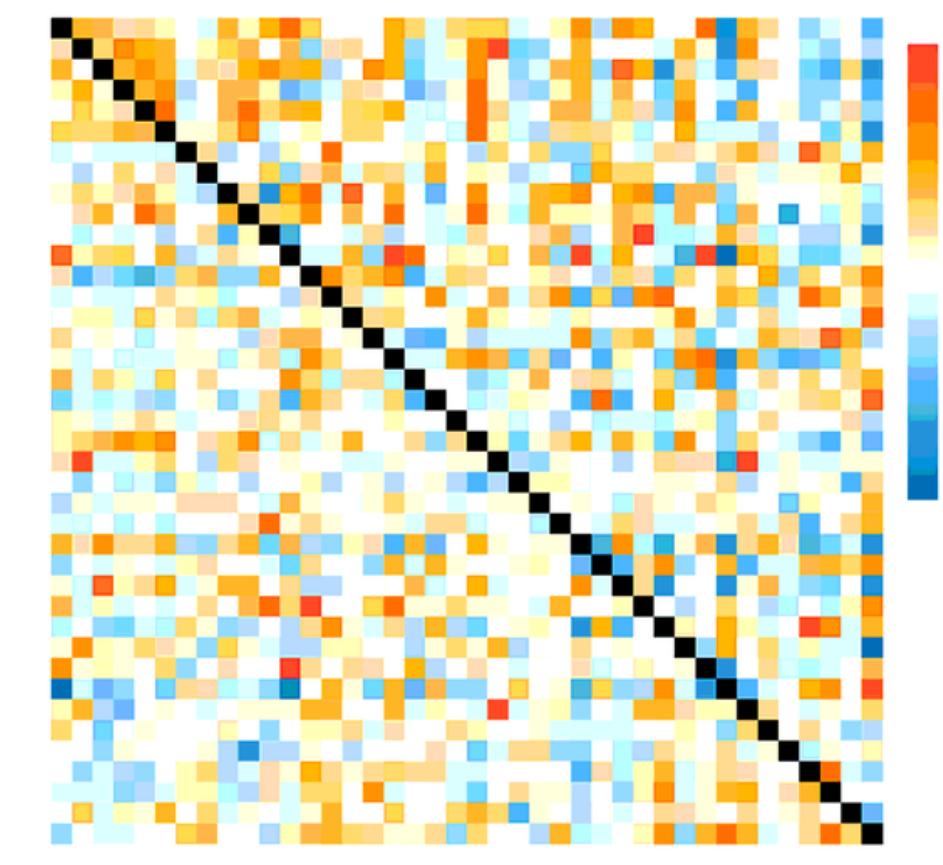

C

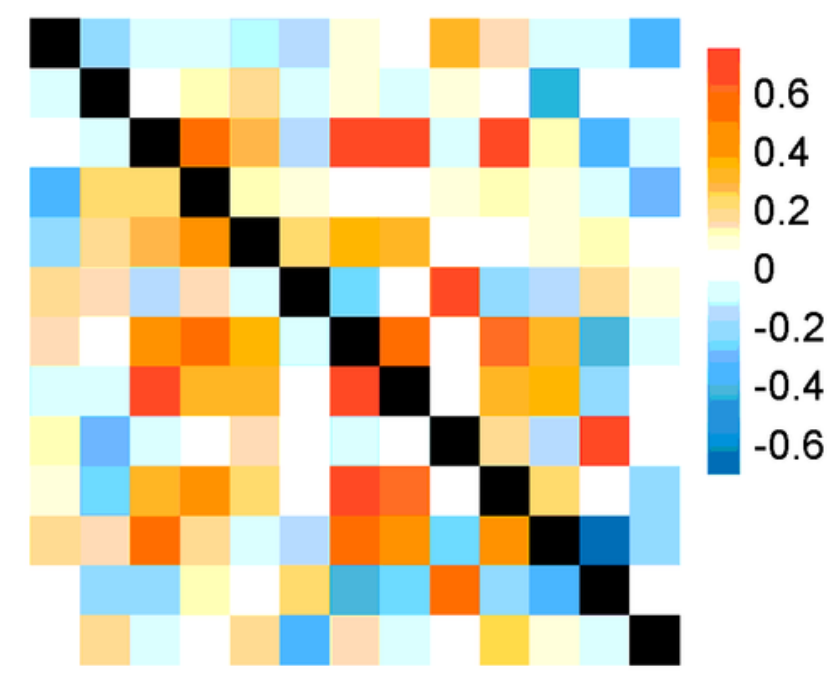

b

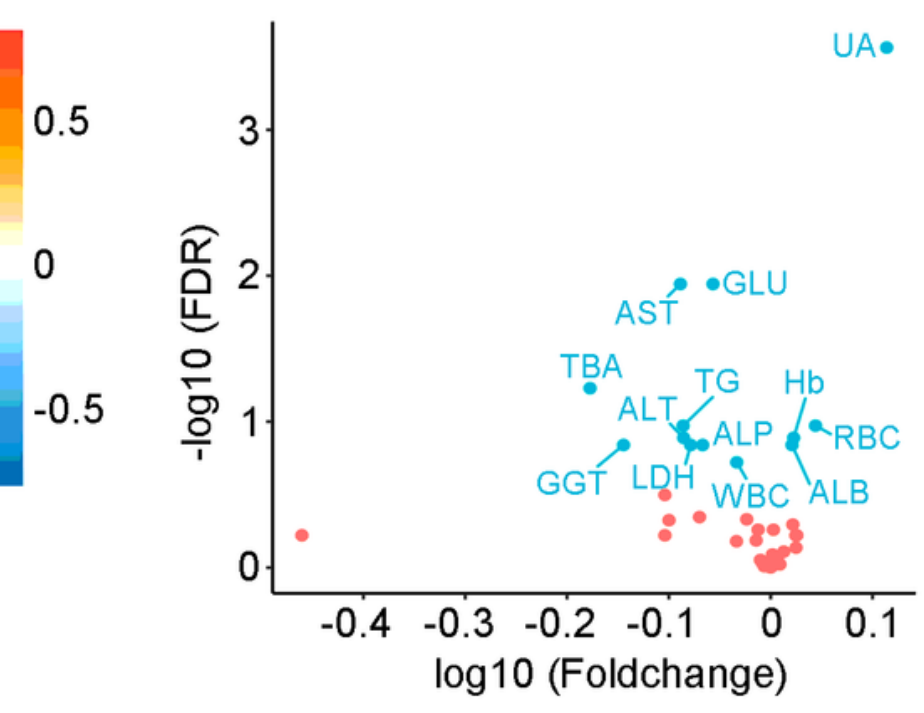

d
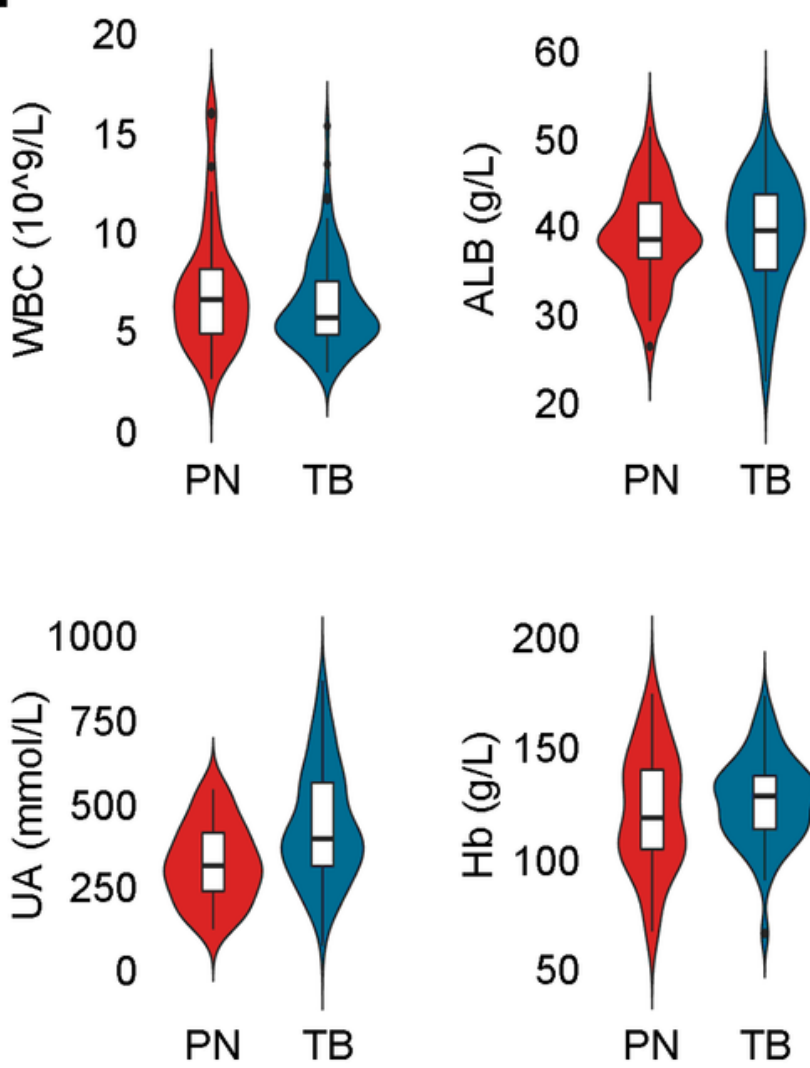

Figure 2

Statistical comparison and multivariate risk model between AFB- IGRA+ TB and PN. A, Comparative differential correlation heatmap. The upper diagonal of the main matrix shows a correlation between pairs of proteins among the PN. The lower diagonal of the heatmap shows a correlation between the same protein pairs in the AFB- IGRA+ TB. Red, positive correlation. Blue, negative correlation. B, Volcano plot showing the distribution of all laboratory variables between the TB and PN. C, Heatmap of pairwise 
significant variables correlation (z-score) in TB and PN. The upper diagonal: PN. The lower diagonal: TB. D, Violin plots showing the values of variables between two groups.

a

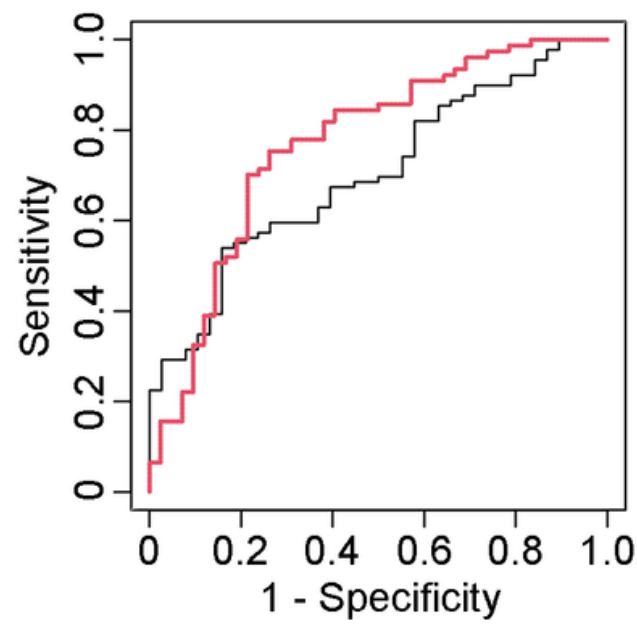

C

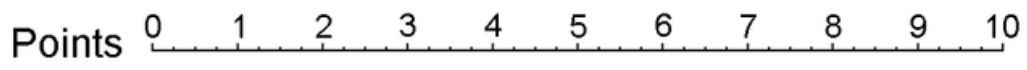

Age $10^{\prime} \quad 30^{\prime} 50^{\prime} 70^{\prime} 90$

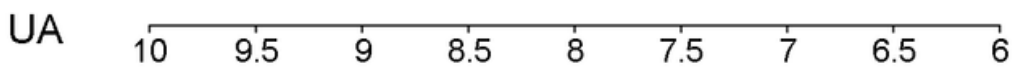

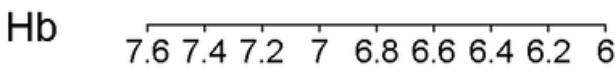

WBC

$\begin{array}{llllllllll}\text { ALB } & 4.2 & 4.4 & 4.6 & 4.8 & 5 & 5.2 & 5.4 & 5.6 & 5.8\end{array}$

$\begin{array}{lllllllllllll}\text { Total } & & & & & & \end{array}$

Probability

$0.1 \quad 0.3 \quad 0.50 .60 .70 .8$ b

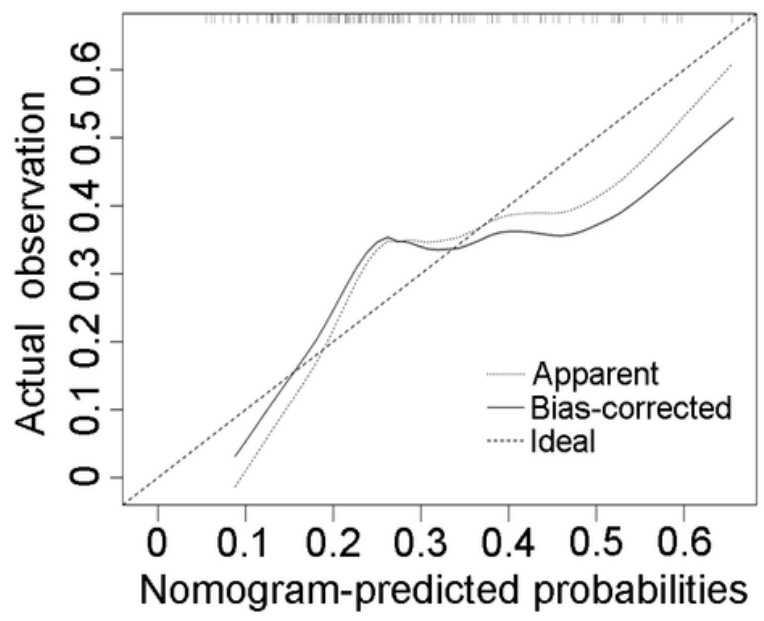

d

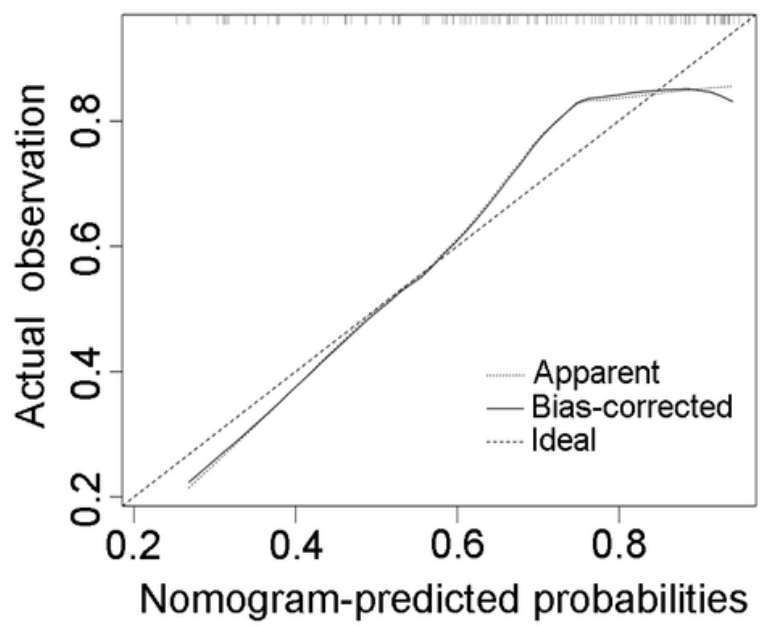

Figure 3

Multivariate risk model to predict PTB probability. A, ROC curves of multivariate risk model and external validation cohort. Black line, multivariate risk model. Red line, external validation cohort. B, Calibration curve of the multivariate risk model. The dotted line represented the ideal fit; circles represented nomogram-predicted probabilities; and solid line represented bias-corrected nomogram-predicted probabilities. C, Nomogram predicting risk based on five variables. Total point values were independently calculated for each cause of outcome and then applied to the corresponding probability scale at the bottom. Values of four variables (UA, Hb, WBC and ALB) were log2-transformed. D, Calibration curve of external validation cohort using nomogram. 


\section{Supplementary Files}

This is a list of supplementary files associated with this preprint. Click to download.

- Supplementaryinformation.xlsx 